\title{
Enhancing thoracic surgical trainee competence in the coronavirus disease 2019 (COVID-19) era: Challenges and opportunities for mentorship
}

\author{
Marko T. Boskovski, MD, MHS, MPH, ${ }^{a}$ Sameer A. Hirji, MD, MPH, ${ }^{a}$ Alexander A. Brescia, MD, MSc, ${ }^{b}$ \\ Andrew C. Chang, $\mathrm{MD},{ }^{\mathrm{c}}$ and Tsuyoshi Kaneko, $\mathrm{MD}^{\mathrm{a}}$
}

The coronavirus disease 2019 (COVID-19) pandemic, caused by severe acute respiratory syndrome coronavirus 2 (SARS-CoV-2), has had a major disruptive social, economic, and health impact globally and has affected more than 1.5 million people in the United States and more than 5 million worldwide so far. ${ }^{1}$ The pandemic has also impacted hospital operations and consequently thoracic surgery (TS) training (including adult cardiac, congenital, and general thoracic) to varying degrees throughout the United States. Although COVID-19 cases are decreasing in some areas, they continue to occupy the capacity of hospitals. Most elective cardiac and general thoracic procedures remain postponed, with priority given to urgent and emergent procedures per guidance and recommendations from the Society of Thoracic Surgeons. ${ }^{2}$ The substantial reduction in surgical case volume during the COVID-19 pandemic has created challenges for the training of TS residents and fellows. Herein, we discuss these challenges, provide a framework for identifying trainees who are most at risk for being impacted, and propose possible solutions to enhance and/or sustain surgical education during the COVID-19 pandemic.

The aggregate impact of the COVID-19 pandemic on TS education depends primarily on 5 key factors, which should be considered in the context of multiple training pathways (independent $5+2$ and $5+3$, fast-track $4+3$, and integrated 6-year programs), as well as the asymmetric vertical nature of most programs whereby the most significant operative experience is emphasized toward the end of residency. These factors include: (1) the overall

From the ${ }^{a}$ Division of Cardiac Surgery, Department of Surgery, Brigham and Women's Hospital, Harvard Medical School, Boston, Mass; and ${ }^{\mathrm{b}}$ Department of Cardiac Surgery and ${ }^{c}$ Section of Thoracic Surgery, Department of Surgery, University of Michigan, Ann Arbor, Mich.

Dr Brescia is supported by the National Research Service Award postdoctoral fellowship (no. 5T32HL076123).

Drs Boskovski and Hirji contributed equally to this article.

Received for publication May 1, 2020; revisions received June 13, 2020; accepted for publication June 15, 2020; available ahead of print June 26, 2020.

Address for reprints: Tsuyoshi Kaneko, MD, Division of Cardiac Surgery, Brigham and Women's Hospital, 15 Francis St, Boston, MA 02115 (E-mail: tkaneko2@ partners.org).

J Thorac Cardiovasc Surg 2020;160:1126-9

$0022-5223 / \$ 36.00$

Copyright (c) 2020 by The American Association for Thoracic Surgery

https://doi.org/10.1016/j.jtcvs.2020.06.062

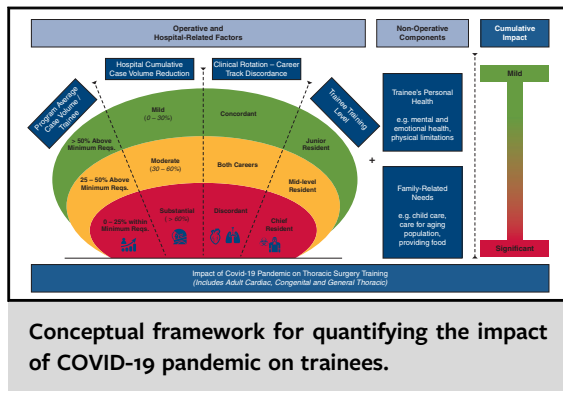

CENTRAL MESSAGE

COVID-19 has created unique challenges to thoracic surgery education. By identifying affected trainees and enhancing existing mentorship models, we can mitigate the negative effects of the pandemic.

See Commentaries on pages 1130 and 1131.

impact of COVID-19 on the trainee's institution, (2) the baseline case volume of the training program, (3) the seniority of the trainee, (4) the concordance or discordance between the trainee's rotations during COVID-19 and their ultimate career track, and (5) the nonoperative components that impact a trainee's ability to learn during the pandemic (Figure 1). These nonoperative factors, which include whether the trainee has been affected by COVID-19, had to take care of sick family members, or provide childcare while schools and daycares are closed, can also have significant effects on education.

We hypothesize that senior trainees (either in a traditional 2- or 3-year fellowship, or in an integrated 6-year program) expected to graduate this year or next, training at lower-volume institutions and/or institutions significantly impacted by COVID-19, on rotations concordant with their career track are most "at risk" of being significantly affected by the pandemic. The impact is most significant for senior trainees for 3 key reasons. First, they may have insufficient case volume to meet existing certification requirements by the American Board of Thoracic Surgery (ABTS) without 


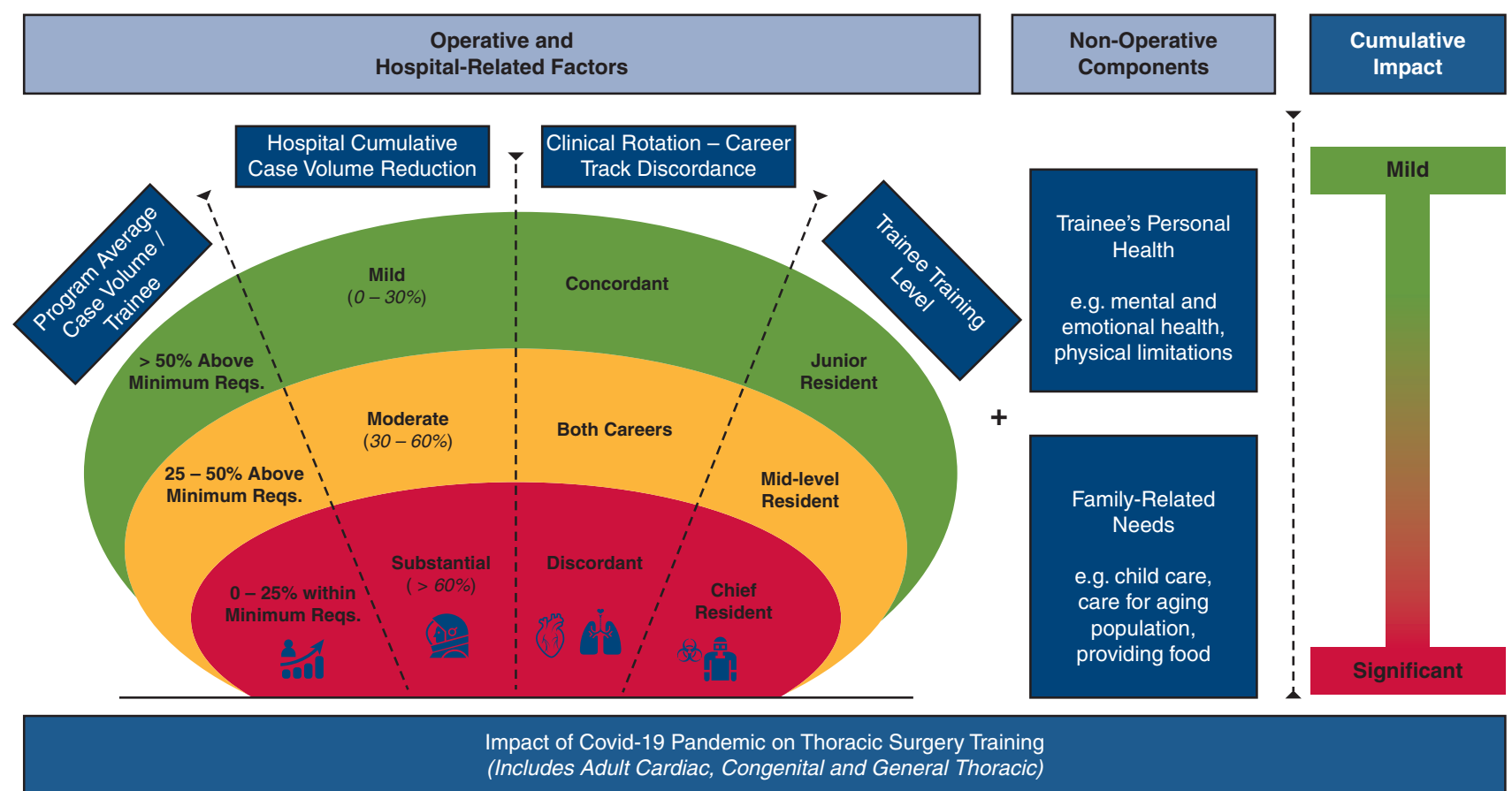

FIGURE 1. Conceptual framework for quantifying the impact of COVID-19 pandemic on TS education. The impact of the COVID-19 pandemic depends primarily on (1) the baseline case volume of the training program, (2) the overall impact of COVID-19 on the trainee's institution, (3) the concordance or discordance between the trainee's rotations during COVID-19 and their ultimate career track, and (4) the seniority of the trainee. The aggregate effect is then assessed visually using the adjacent vertical severity spectrum. COVID-19, Coronavirus disease 2019; TS, thoracic surgery.

an opportunity to make up these cases later in training. Second, trainees typically perform the most cases in their latter part of training - several months of lost operative time has a greater impact for a graduating trainee who would have otherwise been in the operating room most days compared with a junior resident who may have been expected to do a handful of cases in the same time period. Third, for senior trainees there is focused attention not only on technical procedural competence but also cognitive skills (eg, nontechnical skills) and nuanced decision-making (eg, conduct of the operation) that is most often consolidated and impactful toward the latter end of the training. These skills are more difficult to augment by online learning or simulation and are most valuable as senior trainees develop greater autonomy toward becoming independent surgeons.

To mitigate some of these issues, the ABTS, the Accreditation Council for Graduate Medical Education (ACGME), the American Board of Medical Specialties (ABMS), and the Thoracic Surgery Directors Association recently acknowledged the impact of COVID-19 on surgical education, specifically case log requirements. The ACGME and the ABMS rely on case logs to ensure sufficient volume and variety of cases for accreditation of training programs and do not require that case log minima be used as a surrogate for procedural competence of individual graduates. ${ }^{3}$ Instead, it is the responsibility of ACGME-accredited programs to graduate only those trainees who have demonstrated the ability to adequately perform surgical procedures considered essential for their area of practice. A given trainee who has not met all case minima may be deemed by the program director to be surgically competent and be allowed to complete the program. ${ }^{4}$ However, the ABTS has traditionally required trainees to meet case volume and case distribution requirements to be eligible for certification. In the face of the COVID-19 pandemic, the ABTS has asked programs and their candidates at risk of not meeting minimum case requirements to consult with the board regarding whether they would be eligible for entry into the certification process. ${ }^{5}$ Practically speaking, we interpret this guidance to mean that technically competent trainees who are just shy of meeting minimum case requirements due to the COVID-19 pandemic will likely be allowed to sit for the boards. It is particularly important in such situations that the program director and the program's Clinical Competence Committee ensure that the trainee is sufficiently prepared to enter clinical practice.

Several potential scenarios arise from the regulatory framework established by the ABTS, Thoracic Surgery Directors Association, ACGME, and ABMS. First, in a worst-case scenario, trainees whose case logs are deemed insufficient by the ABTS, or whose clinical competence is judged to be inadequate by the program, will require additional training time to demonstrate competency. At 
TABLE 1. Recommendations and specific examples of ongoing initiatives to maximize thoracic surgery resident education during the COVID-19 era

\section{Recommendations}

1. Increase the autonomy of senior residents, and while case volumes are decreased, encourage "double-scrubbing" by junior residents, especially during the recovery phase. Observing surgeries is an often underappreciated exercise that can be incredibly valuable.

2. Include residents in virtual patient clinic visits, which can provide crucial learning for patient selection and shared decision-making.

3. Directly involve residents in the management of patients with COVID-19 in the ICU requiring advanced mechanical circulatory support.

4. Perform critical case reviews of operative videos. We have found these to be particularly helpful as faculty discuss surgical nuances that are not readily available in textbooks and can be difficult to pick up during actual cases.

5. Establish debate-style case-based discussions using online meeting tools (eg, Zoom) to promote use of evidence-based guidelines for patient care.

6. Encourage simulation-based learning including small-and large-vessel anastomosis trainers, deep chest models, as well as laparoscopic, thoracoscopic, and robotic simulators.

\section{Examples of ongoing initiatives/activities}

- Junior residents double-scrub cases during the recovery phase

- Wherever appropriate, have 2 trainees (Senior and Junior) independently perform parts or all of a case

- Compartmentalize the case so that multiple residents can participate in the case.

- Senior residents participated in virtual clinic visits with attending physicians

- Junior and senior residents were assigned to critical care teams for ventilator mechanical circulatory support management, including cannula placement

- Review operative videos from CTSNet with faculty on a weekly basis (eg, mitral valve sizing, prosthesis selection, aortic root replacement, root enlargement, etc)

- Review YouTube videos and other operative videos on lobectomy, segmentectomy, mediastinal dissection

- Two cardiac chief residents debating between transcatheter vs surgical aortic valve replacement in a young, low-risk patient with a bicuspid aortic valve

- Two general thoracic chief residents discussing neoadjuvant vs adjuvant treatment for a patient with a right upper lobe non-small cell lung cancer

- Self-guided robotic curriculum through the Mimic da Vinci Skills Simulator (www.mimicsimulation.com/da-vinci-skills-simulator)

- Use of the vessel anastomosis simulation modules commissioned by the TSDA, developed by The Chamberlain Group, and supported by Medtronic. (https://tsda.org/the-tsda/tsda-vessel-anastomosissimulation-module/)

- Deep chest models and vessel holders developed by The Chamberlain Group

- Esophagogastroduodenoscopy, diagnostic and interventional bronchoscopy, and transthoracic echocardiography simulators

- Laparoscopic skills trainers and video-assisted thoracic surgery (VATS) lobectomy simulator

7. Develop enhanced didactic schedules with particular emphasis on imaging skills such as echocardiography, cardiac catheterization, CT scan assessment, as well as case-based virtual didactic sessions focused on preparation for the ABTS certification examination.

8. Formalize a shared-mentorship model framework between faculty and residents to help establish additional research, quality improvement, or patient safety projects that may aid professional development.

9. Participation in online meetings and courses that have no registration fee and are available for review on-demand.
- An extensive case-based virtual didactic curriculum with an expert faculty moderator and level-appropriate oral board style questions for junior and senior residents

- Weekly joint multidisciplinary rounds with cardiology faculty and fellows

- Each resident meet with a designated faculty member to discuss research opportunities or quality improvement initiatives and participate in weekly research outcomes groups' virtual meetings.
- Review lectures and sessions from conferences available online, including the STS, ACC, AATS, TCT, and others

- Participate in resident Zoom events and podcasts organized by the TSRA, the STS COVID webinars, and featured content including skills courses on CTSNet

COVID-19, Coronavirus disease 2019; ICU, intensive care unit; TSDA, Thoracic Surgery Directors Association; $C T$, computed tomography; $A B T S$, American Board of Thoracic Surgery; STS, Society of Thoracic Surgeons; ACC, American College of Cardiology; AATS, American Association for Thoracic Surgery; $T C T$, Transcatheter Cardiovascular Therapeutics; TSRA, Thoracic Surgery Residents Association. 
large-volume centers, additional training could be accommodated with relative ease. However, at lower-volume institutions, this would present a logistical challenge, as more junior trainees would also have to make up for the operative experience lost during the pandemic, without affecting their future board eligibility requirements.

Even in normal times, the transition from training to independent practice is a challenging period for all thoracic surgeons. ACGME-accredited training and the ABTS certification process ensure that graduates can appropriately handle a wide breadth of cases. Despite this, young surgeons often encounter challenging situations that require guidance and expertise from more experienced colleagues, making strong mentorship critical in this early transition period. For prospective residency program graduates whose operative experience may have been diminished due to the COVID-19 pandemic, mentorship will carry increased importance and should take even greater priority when considering job opportunities. Seasoned mentors for these graduates must recognize the "lost opportunity" and offer broad support to assure a smooth transition into practice. This includes providing not only clinical, but also nonclinical mentorship with attention to coaching for leadership and communication skills, identifying areas for scholarship, nurturing relationships with their own family, and developing mechanisms to reduce mental fatigue and burnout. In cases in which strong mentorship is unavailable for graduating residents at their first job, consider extending training for an additional 6 or 12 months either within the same program or as an apprenticeship in a new program.

As hospitals continue to care for patients with COVID-19 and also slowly return to normal activity levels, there are several initiatives that can be implemented to maximize the education of TS residents. These practical recommendations are highlighted in Table 1, where we also provide specific examples of initiatives undertaken at our 2 institutions. We do not underestimate the importance of the pandemic's effect on junior trainees' education. Given the evolving nature of academic needs during the pandemic, there also likely will be a domino effect for the junior residents, since index cases may be claimed by the sub-chiefs to accommodate the lower case volumes. Additionally, rotations that can be sacrificed to achieve satisfactory operative experience are likely to be adjunct, nonoperative rotations (eg, interventional bronchoscopy, complex gastrointestinal, and cardiac catheterization). While the more junior trainees may not have as large of an impact on operative volume, any impact that they experience may present greater challenges because these are the individuals who are unlikely to get exceptions from the ABTS. Thus, we must maximize the junior resident/fellow's experience by double-scrubbing with senior or compartmentalizing the case to multiple residents. In addition, we must take every opportunity to reinstate these adjunct rotations since these are really important skillsets for all of residents/fellows.

The COVID-19 pandemic presents great challenges to our profession. As residency programs have adapted to institutional and societal needs in responding to these challenges, there has been a dramatic reduction in operative volume, which among other effects has negatively impacted the operative experience of TS trainees, albeit to varying degrees at different institutions. By appropriately identifying trainees who are most affected, and enhancing existing and/or establishing new mentorship models, we can recognize and mitigate some of the negative effects of the pandemic on surgical education to continue to produce safe, well-trained, and competent thoracic surgeons. Ongoing mentorship will also undoubtedly remain integral to early career development in the post-COVID era, particularly so for this graduating class.

\section{Conflict of Interest Statement}

Dr Kaneko reported consultant/speaker for Edwards Lifesciences, Medtronic, Abbott Structural Heart, Baylis Medical, and 4C Medical. All other authors reported no conflicts of interest.

The Journal policy requires editors and reviewers to disclose conflicts of interest and to decline handling or reviewing manuscripts for which they may have a conflict of interest. The editors and reviewers of this article have no conflicts of interest.

\section{References}

1. COVID-19 Dashboard; 2020. Available at: https://coronavirus.jhu.edu/map.html. Accessed April 22, 2020.

2. Haft JW, Atluri P, Alawadi G, Engelman D, Grant MC, Hassan A, et al. Adult cardiac surgery during the COVID-19 pandemic: a tiered patient triage guidance statement. Ann Thorac Surg. April 10, 2020 [Epub ahead of print].

3. The American Board of Medical Specialties and Accreditation Council for Graduate Medical Education Joint Principles: Physician Training During the Coronavirus Disease 2019 Pandemic; 2020. Available at: https://acgme.org/Newsroom/ Newsroom-Details/ArticleID/10192/The-American-Board-of-Medical-Specialtiesand-Accreditation-Council-for-Graduate-Medical-Education-Joint-PrinciplesPhysician-Training-During-the-Coronavirus-Disease-2019-Pandemic. Accessed April 22, 2020.

4. Potts JR. COVID-19: Special Communication to Surgical Program Directors, including Case Log Guidance; 2020. Available at: https://www.acgme.org/Portals /0/Documents/COVID-19/SurgCOVID19LTC.pdf. Accessed April 22, 2020.

5. Putnam JB, Fullerton DA. Impact of COVID-19 pandemic on ABTS case requirements and optional ABTS case requirements' review; 2020. Available at: https://tsda.org/wp-content/uploads/2020/04/ABTSLetterToPDAndResidents COVIDFINALdocx.pdf. Accessed April 22, 2020. 